\title{
Therapie des inguinalen Crossenrezidivs: Ist die offene Re-Crossektomie noch zeitgemäß?
}

\section{Treatment of recurrent saphenofemoral incompetence: surgical approach still up-to-date?}

Autoren

Stefania Aglaia Gerontopoulou' ${ }^{1}$, Knuth Rass ${ }^{2}$

Institute

1 Universitätsklinikum Hamburg-Eppendorf, Klinik für Dermatologie und Venerologie

2 Eifelklinik St. Brigida, Zentrum für Venen und periphere Arterien

Schlüsselwörter

Crossenrevision, endovenös thermische Ablation, Rezidivvarikose, Verödung

Key words

Endothermal ablation, inguinal reoperation, recurrent varicose veins, sclerotherapy

eingereicht $07.10: 2018$

akzeptiert $\quad 18.11 .2018$

Bibliografie

DOI https://doi.org/10.1055/a-0800-9183

Phlebologie 2019; 48: 39-46

(c) Georg Thieme Verlag KG Stuttgart · New York ISSN 0939-978X

Korrespondenzadresse

Priv.-Doz. Dr. med. Knuth Rass

Zentrum für Venen und periphere Arterien

Eifelklinik St. Brigida GmbH \& Co. KG

Kammerbruchstr. 8

52152 Simmerath

Tel.: 02473/89-2325

Fax: 02473/89-2440

E-Mail: knuth.rass@st-brigida.de

\section{ZUSAMMENFASSUNG}

Einleitung Die Rezidivvarikose ist trotz der Entwicklung neuer Verfahren und Operationsstrategien ein relevantes medizinisches Problem. Zur Therapie inguinaler Crossenrezidive (ICR) steht die offene Re-Crossektomie zur Verfügung, die derzeit gegenüber weniger invasiven Verfahren in den Hintergrund tritt. Methoden Diese Übersichtsarbeit basiert auf aktuell verfügbaren Leitlinien und einer Literaturrecherche bei PubMed zur Frage des Einsatzes und der Effektivität verschiedener Verfahren zur Therapie des ICR.

Ergebnisse Inguinale Re-Crossektomie und Schaumsklerosierung sind uneingeschränkt anwendbar. Die Operation, sofern mit Barrieretechnik kombiniert, scheint langfristig effektiver als die Verödung und ist mit einer hohen Patientenzufriedenheit verbunden. Endovenös thermische Ablationsverfahren können für individuelle Fälle mit geeignetem anatomischem Befund eingesetzt werden. Vergleichende Studien fehlen.

Schlussfolgerung Die offene Re-Crossektomie ist zeitgemäß. Ob der Vorteil der potentiell höheren Effektivität bei der Operation stärker wiegt als die geringere Invasivität der Schaumsklerosierung verbunden mit häufigeren Therapiesitzungen müsste mittels randomisierter kontrollierter Studien geklärt werden.

\section{ABSTRACT}

Introduction Recurrent varicose veins are a common medical problem despite the development of new operation techniques and a variety of minimal invasive treatments. The ideal treatment of recurrent saphenofemoral incompetence is still matter of debate. Nowadays, the open inguinal reoperation seems to fade into the background because of available less invasive treatment alternatives.

Methods This review article is based on current guidelines and a systematic PubMed search of article references dealing with the use and effectiveness of various techniques for the treatment of recurrent saphenofemoral incompetence. Besides this main topic, we highlight the causes, classification and frequency of recurrence after varicose vein treatment.

Results Recurrence of varicose veins after surgical or endovenous treatment for great saphenous vein incompetence is a frequent event. Causes for same site recurrence are neovascularization, especially after surgical treatment, and recanalization or reflux into groin tributaries after endovenous ablation leaving a saphenofemoral stump, as well as tactical and technical errors. Disease progression is leading to different site recurrence. Clinically relevant saphenofemoral recurrence can be treated by inguinal reoperation or ultrasound guided foam sclerotherapy without any anatomical restrictions. However, inguinal reoperation particularly when providing a barrier technique seems to be more effective than sclerotherapy in the long-term and is connected with a high patients' satisfaction in spite of its invasiveness. Endovenous ablation is likewise effective but limited to the treatment of persistent saphenous 
or accessory trunks. In many cases the combination of surgical or endovenous treatment with foam sclerotherapy is a feasible approach.

Conclusion Due to its long-term efficacy the surgical approach with inguinal reoperation for recurrent saphenofemoral incompetence is indeed still up-to-date. Randomized studies comparing surgery, endovenous ablation and foam sclerotherapy are needed to find out, if the potentially lower recurrence rates of the surgical approach outweigh the lower invasiveness with a need for repeated treatment sessions in case of sclerotherapy.

\section{Einleitung}

Die Varikose und die damit einhergehende chronisch venöse Insuffizienz (CVI) gehören mit einer Prävalenz von über 30 \% bei Erwachsenen zu den häufigsten Erkrankungen in Industrieländern [1]. Sie führen im Krankheitsverlauf zu einer relevanten Beeinträchtigung der Lebensqualität [2], zu chronischen Entzündungen, Ulzerationen, und erhöhen signifikant das Thrombembolierisiko [3], so dass in Leitlinien zu frühzeitiger aktiver Behandlung geraten wird $[4,5,6]$. Bei Vorliegen einer Stammveneninsuffizienz sollte dem Patienten ein endovenös-ablatives Verfahren, z. B. endovenöse Laseroder Radiofrequenzablation, oder die offene Operation mit Crossektomie und Stripping angeboten und die aktive Therapie gegenüber einer alleinigen Kompressionstherapie bevorzugt werden [6].

Weniger eindeutig sind die Empfehlungen bei Auftreten einer Rezidivvarikose, die ihren Ursprung im Bereich der zuvor behandelten Crossenregion hat. Wie nicht nur in aktuellen randomisierten kontrollierten Studien gezeigt werden konnte, treten klinische Rezidive sowohl nach offener Operation, als auch nach endovenöser Ablation fünf Jahre nach einer Behandlung in ca. 50 \% der Fälle mit unterschiedlich starker Ausprägung auf [7]. Diese klinischen Rezidive sind in der Regel definiert nach den Kriterien der REVAS-Klassifikation (Recurrent varices after surgery): Vorhandensein von Varizen an unteren Extremitäten, die zuvor bereits wegen Krampfadern operiert wurden. Diese klinische Definition umfasst das „echte Rezidiv“, welches sich im Operationsgebiet entwickelt, aber auch verbliebene, präoperativ bereits diagnostizierte Varizen, sowie neu entstandene Varizen als Konsequenz des fortschreitenden Krankheitsprozesses [8]. Einen relevanten Anteil der klinischen Rezidive stellen im Langzeitverlauf (5 Jahre postoperativ) diejenigen, die der zuvor behandelten Crossenregion entstammen, 18-33\% nach endovenöser Laserablation der V. saphena magna (VSM), 5-17\% nach inguinaler Crossektomie und Stripping der VSM, jeweils bezogen auf die Gesamtheit der behandelten Patienten [9]. Die duplexsonograpisch nachweisbaren Crossenrezidive unterscheiden sich offenbar verfahrensabhängig: Während nach korrekt durchgeführter Crossektomie Rezidive vorwiegend durch eine Neovaskularisation im saphenofemoralen Gebiet entstehen, spielen nach Thermoablation eher Rekanalisierungen und Neorefluxe über Crossenseitenäste die wesentliche Rolle für die spätere Entwicklung eines „echten“ klinischen Rezidivs [10,11].

Neben den „unvermeidlichen“ Rezidiven, die trotz sorgfältiger Operations- bzw. Behandlungstechnik auftreten, sind Rezidive aufgrund von technischen Fehlern (z. B. Belassen eines saphenofemoralen Mündungsstumpfes) oder auch taktischen Fehleinschätzungen (diagnostische Fehler) zahlenmäßig vermutlich ebenso relevant, zumindest soweit es die Indikation für einen Revisionseingriff betrifft [12].
Die offen-chirurgische Crossenrevision für die Behandlung des inguinalen Crossenrezidivs, insbesondere bei Vorhandensein eines Stumpfes und klinisch relevanter Rezidivvarikose, gilt zwar als Referenzmethode, ist aber technisch anspruchsvoll und weist ein im Vergleich zur primären Crossektomie höheres Komplikationsrisiko auf [4]. Die zusätzliche Anwendung von Barrieretechniken in den letzten Jahren ist eine vielversprechende Entwicklung um ein erneutes Crossen-Re-Rezidiv zu verhindern [13,14,15]. Zunehmend werden gerade aufgrund des Komplikationsrisikos weniger invasive Methoden empfohlen und eingesetzt, wie die ultraschallgesteuerte Schaumsklerosierung (UGSS) $[6,16]$. Die Kombination von Operation und UGSS oder Schaumsklerosierung in situ durch Direktpunktion stellt eine weitere, häufig geübte Behandlungspraxis dar [17].

Somit ergeben sich viele offene Fragen zur Therapie des inguinalen Crossenrezidivs: Wenn ein Behandlungsbedarf besteht, welche ist die geeignetste Methode? Ist die Auswahl des Verfahrens befundabhängig? Welche methodenabhängigen Vor- und Nachteile gibt es? Werden weniger invasive Methoden an Bedeutung gewinnen? Ist die offene Re-Crossektomie aufgrund der Invasivität überhaupt noch zeitgemäß?

Diese Übersichtsarbeit basiert auf den aktuell verfügbaren Leitlinien und auf neuerer Literatur zur Frage der Genese, klinischen Relevanz und Behandlungsmöglichkeiten des inguinalen Crossenrezidivs.

\section{Definition und Ätiopathogenese der Rezidivvarikose}

Es existieren unterschiedliche Definitionen und Klassifikationen für die Rezidivvarikose.

Im Jahr 2000 wurde durch Michel Perrin die REVAS-Klassifikation inauguriert, die zunehmend im Rahmen von prospektiven Studien, auch zur Rezidivchirurgie, eingesetzt wird und sich als Standard für die Erfassung klinischer Rezidive etabliert hat $[8,18]$. Dabei lassen sich Rezidivvarizen wie folgt differenzieren:

1. Residual-Varizen, die vor einem geplanten Eingriff dokumentiert wurden und postoperativ persistieren,

2. neue aufgetretene Varizen in einem bislang nicht behandelten Areal als Folge der Progression der Grunderkrankung und schließlich

3. „echte“ Rezidivvarizen („true recurrence“), die als Folge einer Neovaskularisation oder infolge von technischen oder taktischen Fehlern auftreten.

Um die REVAS-Klassifikation auf endovenöse Therapieverfahren ebenfalls anwenden und neben der Neovaskularisation auch Re- 
kanalisierungen und Neorefluxe über Crossenseitenäste, z. B. die V. saphena accessoria anterior (VSAA), erfassen zu können, wurde durch unsere Arbeitsgruppe eine modifizierte REVAS-Klassifikation vorgeschlagen [7].

Die Rezidivvarikose wird nach klinischen Kriterien und, zur Frage der Genese und des Ursprungs, durch eine duplexsonographische Diagnostik klassifiziert $[6,16]$. Nach UIP-Konsensus werden unterschieden [19]:

1. Rezidive über einen belassenen Stumpf: Wenn im Rahmen der inguinalen Crossektomie die Ligatur der saphenofemoralen Junktion nicht niveaugleich an der V. femoralis communis erfolgt, sondern weiter distal, entsteht ein Stumpf, wie es zumeist auch nach endovenösen Ablationsverfahren methodenimmanent der Fall ist („distaler Verschlusstyp“). Aus diesem Stumpf können sich über präexistente Seitenäste oder Neovaskulate klinische Rezidive entwickeln, nicht selten unter Einbeziehung einer insuffizienten VSAA,

2. Rezidive infolge einer Neovaskularisation: Neubildung von kleinkalibrigen Gefäßen in einer voroperierten Region, die sich durch Verbindungen mit der tiefen Vene füllen.

In einer aktuellen Metaanalyse zur Therapie der Stammveneninsuffizienz der VSM wurden randomisierte kontrollierte Studien mit einem Follow-up von mindestens 5 Jahren eingeschlossen. Duplexsonographisch detektierte inguinale Crossenrezidive nach Crossektomie und Stripping, endovenöser Laserablation (EVLA) oder UGSS werden mit Raten von 7-38\% beschrieben, wobei die Rezidivrate nach Crossektomie und Stripping signifikant geringer ist im Vergleich zu EVLA und UGSS (20).

Man kann mindestens vier wichtige Ursachen der Rezidivvarikose benennen:

Die Progression der Grunderkrankung ist eine der wichtigsten Ursachen der Rezidivvarikose. Sie kann sich aszendierend (oberflächliche Venen dilatieren und werden varikös), deszendierend (Ausgangspunkt ist ein Reflux an der saphenofemoralen bzw. -poplitealen Junktion oder ein Reflux aus insuffizienten Perforatoren) oder multifokal entwickeln $[6,18]$. Für die Varikogenese bedeutsam sind u. a. genetische Faktoren, die zu Störungen des Aufbaus der extrazellulären Matrix (Fibulin-3, Matrix-Metalloproteinasen) führen [21]. Dies stellt vermutlich den Ausgangspunkt für die pathologische Venenerweiterung mit konsekutivem Funktionsverlust von Venenklappen dar. Zudem wird diskutiert, dass sich durch Änderung von Druckverhältnissen in Beinvenen, auch beispielsweise infolge therapeutischer Eingriffe, eine Remodellierung der Venenwand mit einem Umbau des elastischen und kollagenen Fasergerüstes und mit Aktivierung bestimmter Matrixmetalloproteinasen (MMP-2, MMP-9) entwickeln kann. In der aktuellen Literatur finden sich vermehrt Hinweise dafür, dass dieses „Remodelling“, das zu einer Dilatation der Venen führt, allein durch einen chronisch bestehenden erhöhten venösen Füllungsdruck, wie z. B. durch lange Steh- oder Sitzbelastung, Schwangerschaft, Bewegungsmangel und Adipositas als typische Realisationsfaktoren der primären Varikosis, induziert werden kann [22]. Die Adipositas ist zudem ein unabhängiger Risikofaktor für die Entstehung einer Rezidivvarikose [23]. Somit kommt der genetischen Veranlagung und dem individuellen Lebensstil sowohl für die Progression der Grunderkran- kung, als auch für die Entwicklung eines „echten“ Rezidivs (s. u.) eine wichtige Bedeutung zu.

Die Neovaskularisation wird als wichtigste Ursache für ein klinisches Rezidiv nach einer fehlerfrei durchgeführten offenen Operation, insbesondere an der saphenofemoralen Junktion, eingeschätzt $[10,18]$. Neovaskularisation ist gekennzeichnet durch die Ausbildung neuer Gefäße, die im Gegensatz zu residuellen Venen histopathologische Unterschiede aufweisen. Hierzu zählt der Verlust der Wandstruktur, das Fehlen von Klappen und Nerven und das Vorhandensein multipler Lumina innerhalb eines vernarbten Bindegewebes [24]. Physikalische intraoperative Faktoren, wie die Art des angewendeten Nahtmaterials in der Crosse, die chirurgische Technik mit Exposition freiliegenden Endothels sowie das operative Trauma zählen zu den vermuteten Auslösern der Neovaskularisation. Postoperative Faktoren, wie die Hypoxie z. B. im Stumpfbereich ligierter Venen, Mechanismen des Wundheilungsprozesses, die veränderte Hämodynamik (Remodelling, s. o.), inflammatorische und mikrothrombotische Ereignisse, sind vermutlich ebenfalls an der Pathogenese der Neovaskularisation beteiligt [25].

Von taktischen Fehlern spricht man, wenn präoperativ keine oder nur eine unzureichende Evaluation des Refluxes stattgefunden hat, insbesondere der oder die proximalen Insuffizienzpunkte nicht korrekt definiert wurden, oder eine für den Befund ungeeignete Behandlungsmethode gewählt wurde [18]. Die Durchführung einer präoperativen Duplexuntersuchung, die heute sicher als obligatorisch anzusehen ist, reduziert die Rate an taktischen Fehlern und verbessert das Therapieergebnis erheblich [26].

Technische Fehler spielen eine wichtige Rolle für das Auftreten einer Rezidivvarikose [12]. Interventionsbedürftige Rezidive sind überwiegend die Folge einer technisch unzureichenden Operation, z. B. durch das Belassen eines langen Saphenastumpfes. Diese Beobachtung unterstreicht die Notwendigkeit einer bündigen („niveaugleichen“) Ligatur der VSM am Übergang zur V. femoralis communis [27]. Technische Fehler lassen sich durch entsprechende Spezialisierung und strukturierte Ausbildung vermeiden.

\section{Therapie des inguinalen Crossenrezidivs}

Für die Behandlung des inguinalen Crossenrezidivs kommen mehrere Therapieverfahren in Betracht: Endovenös thermische Ablationsverfahren, die UGSS, schließlich die offen-chirurgische Crossenrevision sowie die Kombination der Verfahren. Aufgrund des höheren Schwierigkeitsgrades und Komplikationsrisikos der Crossenrevision im Vergleich zur primären Operation, ist ein Trend hin zu den weniger invasiven Methoden zu beobachten $[6,16]$. Auswahl und Einsatz der unterschiedlichen Verfahren hängt maßgeblich von den vorliegenden Befunden ab und sollte präoperativ anhand des klinischen und duplexsonographischen Bildes unter Berücksichtigung des Patientenwunsches festgelegt werden ( $\triangleright \mathbf{A b b} \mathbf{1} \mathbf{1})$.

\section{Endovenös thermische Ablationsverfahren (EVTA)}

Die EVTA sind nicht für alle Formen der Rezidivvarikose geeignet $[28,29]$. Rezidivbefunde mit persistierenden VSM-Segmenten oder einer gerade verlaufenden VSAA können mit endothermischen Katheterverfahren behandelt werden [29]. Sofern der Katheter bis nahe an den saphenofemoralen Übergang eingebracht werden kann, sind alle verfügbaren Verfahren - EVLA, Radiofrequenz- 


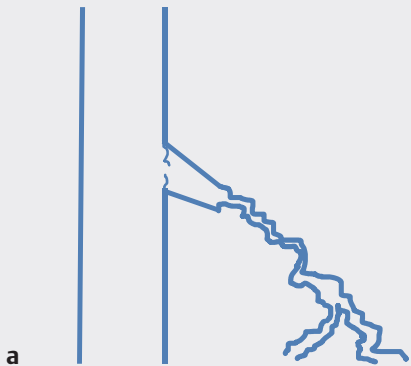

\begin{abstract}
- Abb. 1 Muster saphenofemoraler Crossenrezidive und therapeutische Optionen unter der Voraussetzung einer vorhandenen klinischen Relevanz. a Belassener Crossenstumpf mit Neovaskulaten und Übergang in eine Seitenastvarikose; keine sondierbaren Venenstämme in anatomischer Nähe zur Refluxquelle $\rightarrow$ Therapievorschlag: Inguinale Crossenrevision. b Neovaskularisation mit Übergang in Seitenastvarikose ohne Nachweis eines Crossenstumpfes; keine sondierbaren Venenstämme in anatomischer Nähe zur Refluxquelle $\rightarrow$ Therapievorschlag: Schaumsklerosierung oder inguinale Crossenrevision in Kombination mit Schaumsklerosierung. c1 Neovaskularisation (kurzes Segment) oder kurzer Stumpf mit Übergang in einen sondierbaren Venenstamm (z. B. VSM, VSAA) $\rightarrow$ Therapievorschlag: Endovenös-thermische Ablation oder inguinale Crossenrevision und Exhairese des Venenstamms. c2 Neovaskularisation (längeres Segment) mit Übergang in einen sondierbaren Venenstamm (z. B. VSM, VSAA) $\rightarrow$ Therapievorschlag: Endovenös-thermische Ablation in Kombination mit Schaumsklerosierung oder inguinale Crossenrevision, Schaumsklerosierung und Exhairese.
\end{abstract}
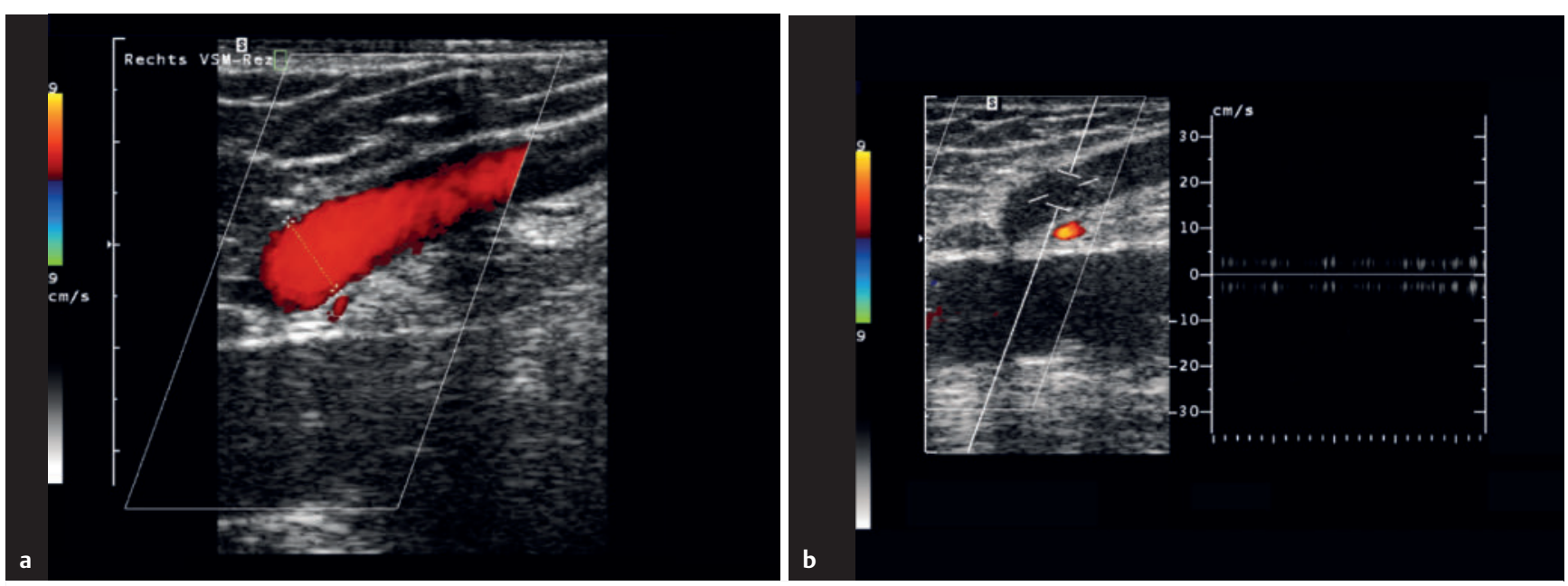

Abb. 2 Duplexsonographische Darstellung eines inguinalen Crossenrezidivs mit vollständig erhaltener insuffizienter V. saphena magna rechts. a Refluxnachweis im Farbdoppler. b 1 Monat nach endovenöser Laserablation Nachweis einer vollständigen Okklusion mittels Farb- und pw-Doppler (kein Reflux bei Durchführung des Valsalva-Manövers).

ablation, Heißdampf - grundsätzlich einsetzbar ( $\triangleright$ Abb. 1 c1 und - Abb. 2). Häufig ist jedoch zwischen Venenstamm und der Mündung in die VFC ein mehr oder weniger langes Segment mit Neovaskulaten zwischengeschaltet. Hier kann beispielsweise der Einsatz von Heißdampf oder eine Kombination von thermischer Ablation und Schaumverödung über den liegenden Katheter, wie es bei dem segmentalen Radiofrequenzkatheter möglich ist, sinnvoll sein ( $\triangleright$ Abb. 1c2 und $\triangleright$ Abb. 3). Eine hohe Patientenzufriedenheit, geringe Komplikationsraten und kurze Operationszeiten lassen die EVTA als attraktive Behandlungsalternative gegenüber der inguinalen Crossenrevision in geeigneten Fällen erscheinen [28]. Studien mit Nachbeobachtungszeiten bis zu 18 Monaten belegen eine Effektivität mit Verschlussraten um die $95 \%$ [29,30]. Langzeitergebnisse im Rahmen der Rezidivbehandlung sowie kontrollierte prospektive Studien fehlen allerdings noch und die EVTA-Verfahren stehen aufgrund fehlender Kostenerstattung im ambulanten Sek- tor nicht jedem Patienten zur Verfügung. Vor- und Nachteile der EVTA gegenüber der inguinalen Crossenrevision sind in $\triangleright$ Tab. 1 zusammengefasst.

\section{Ultraschallgesteuerte Schaumsklerosierung (UGSS)}

Gemäß der europäischen Leitlinie zur Sklerotherapie können alle Varizen, unabhängig von Art und Kaliber, also auch Rezidivvarizen, mittels Verödungstechniken, insbesondere mit der UGSS behandelt werden [31]. Somit ist eine Beschränkung auf bestimmte Befunde, wie bei den EVTA-Verfahren, zunächst nicht gegeben. Der Vorteil der UGSS gegenüber allen anderen Verfahren liegt in der geringen Invasivität und der Kosteneffektivität. Auch wenn es häufig zu duplexsonograpisch nachweisbaren inguinalen Crossen-Re-Rezidiven kommt, so kann die Sklerotherapie doch grundsätzlich beliebig wiederholt werden. Man könnte daher sagen, dass die Sklerotherapie palliativen Charakter hat. Sie ist in bestimmten Situationen 

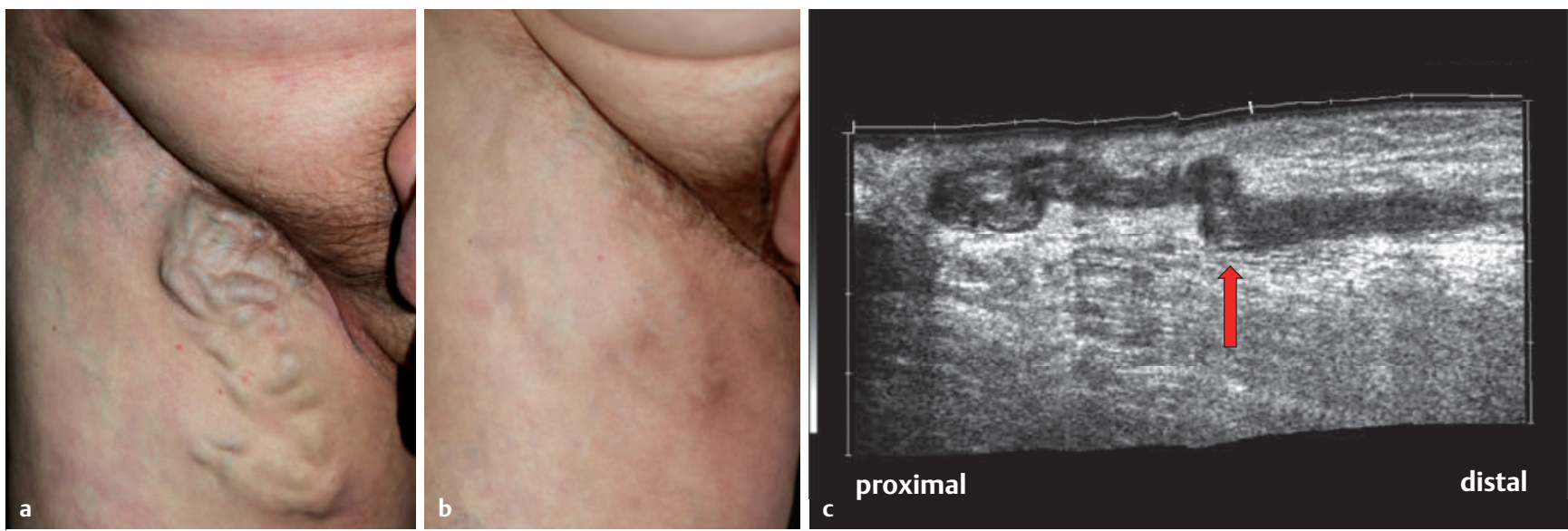

Abb. 3 Ausgedehntes rechts inguinales Crossenrezidiv nach ilioinguinaler Lymphknotendissektion. a Klinischer Aspekt präoperativ. b Klinischer Aspekt 3 Monate nach endovenöser Laserablation einer persistierenden VSM (von $10 \mathrm{~cm}$ unterhalb der Leistenfalte bis Hach IV) in Kombination mit Schaumsklerosierung des inguinalen Konvolutes über den vor der thermischen Ablation eingebrachten Angiographiekatheter. c Die sonographische Kontrolluntersuchung (SieScape ${ }^{\circledR}$ ) am 1. postoperativen Tag zeigt einen vollständigen Verschluss des inguinalen Konvolutes. Der Pfeil weist auf den Übergang zur persistierenden VSM.

\Tab. 1 Übersicht der Vor- und Nachteile der unterschiedlichen Therapiemodalitäten

\begin{tabular}{|c|c|c|c|}
\hline & ICR & EVTA & UGSS \\
\hline Vorteile & $\begin{array}{l}\text { - anwendbar für alle Rezidivarten, } \\
\text { v. a. bei kaliberstarken Mündungssituationen } \\
\text { - langfristige Erfahrung } \\
\text { - gut belegte Effektivität für bestimmte } \\
\text { OP-Techniken (vgl. - Tab. 2) }\end{array}$ & $\begin{array}{l}\text { - } \text { geringes Nachblutungsrisiko } \\
\text { - } \text { weniger invasiv } \\
\text { - } \text { aürzere Arbeitsunfähigkeit } \\
\text { - keine Vollnarkose erforderlich }\end{array}$ & $\begin{array}{l}\text { - kosteneffektiv } \\
\text { - gering invasiv } \\
\text { - wiederholbar } \\
\text { - kombinierbar }\end{array}$ \\
\hline Nachteile & $\begin{array}{l}\text { - } \text { meist mit stationärem Aufenthalt verbunden } \\
\text { - Komplikationsrisiko größer als bei anderen Verfahren } \\
\text { - höherer Schwierigkeitsgrad der Operation, umfang- } \\
\text { reiche Erfahrung notwendig } \\
\text { - zeitaufwändige Operation }\end{array}$ & $\begin{array}{l}\text { - fehlende generelle Kostenerstattung } \\
\text { - anatomische Anwendungslimitationen } \\
\text { - Langzeitergebnisse fehlen }\end{array}$ & $\begin{array}{l}\text { - Thromboserisiko } \\
\text { - Datenlage zur Effektivität } \\
\text { noch unzureichend }\end{array}$ \\
\hline
\end{tabular}

Abkürzungen: EVTA = endovenös thermische Ablationsverfahren; ICR = inguinale Crossenrevision; UCSS = ultraschallgesteuerte Schaumsklerosierung

alternativlos ( $>$ Abb. 3 ). Drei bis fünf Jahre nach UGSS von inguinalen Crossenrezidiven beträgt die duplexsonographische Re-Rezidivrate bei Pavei et al. $28 \%$, verbunden mit einem klinischen ReRezidiv bei $20 \%$ der Patienten [32].

Ein besonderer Stellenwert der Schaumsklerosierung liegt zudem in der Kombinierbarkeit mit EVTA und insbesondere mit der offenen inguinalen Crossenrevision [17].

Dennoch birgt die Sklerotherapie auch Risiken. Beispielsweise kann die direkte Injektion des Sklerosierungsmittels im Stumpfbereich bei inguinalem Crossenrezidiv mit einem erhöhten Thromboserisiko verbunden sein [17]. Daraus kann sich auch eine gewisse Limitierung der UGSS mit zunehmendem Durchmesser des inguinalen Rezidivgefäßes ergeben. Die Notwendigkeit einer oder mehrerer Wiederholungen der Therapie kann ebenfalls nachteilig sein, da viele Patienten eine einmalige Behandlung bevorzugen [33]. Die Datenlage zur Langzeiteffektivität der Sklerotherapie des inguinalen Crossenrezidivs ist insgesamt schwach, valide Aussagen hierzu sind kaum möglich. Vor- und Nachteile der UGSS sind in > Tab. 1 zusammengefasst.

\section{Die inguinale Crossenrevision}

Die operative Therapie mit inguinaler Crossenrevision ist ebenfalls bei jeglicher Form saphenofemoraler Crossenrezidive anwendbar ( $\triangleright$ Abb. 1). Gegenüber EVTA-Verfahren wird sie in einem Verhältnis von ca. 70:30 \% häufiger eingesetzt, wie eine große retrospektive Analyse aus den Niederlanden zeigt [34]. Die Crossenrevision scheint besonders geeignet, um bei kaliberstarken Mündungssituationen z. B. mit belassenem Stumpf ( $\triangleright$ Abb. 1a) eine langfristige saphenofemorale Rezidivfreiheit zu ermöglichen und ist nach eigener Erfahrung in dieser Situation auch kein schwieriger oder komplikationsträchtiger Eingriff [15]. Operationstechnisch sind unterschiedliche Zugangswege zum saphenofemoralen Rezidivursprung beschrieben, wobei sich ein ventraler Zugang bei geringen und ein lateraler Zugang mit Präparation über die Vasa femorales ( $\triangleright$ Abb. 4) bei starken Verwachsungen bewährt haben [15,35].

Dennoch gilt die inguinale Crossenrevision in der weltweiten Literatur durchaus als Herausforderung für den Operateur, verbunden mit einem erhöhten Komplikationsrisiko und entsprechender Einschränkung der postoperativen Aktivität und Lebensqualität 

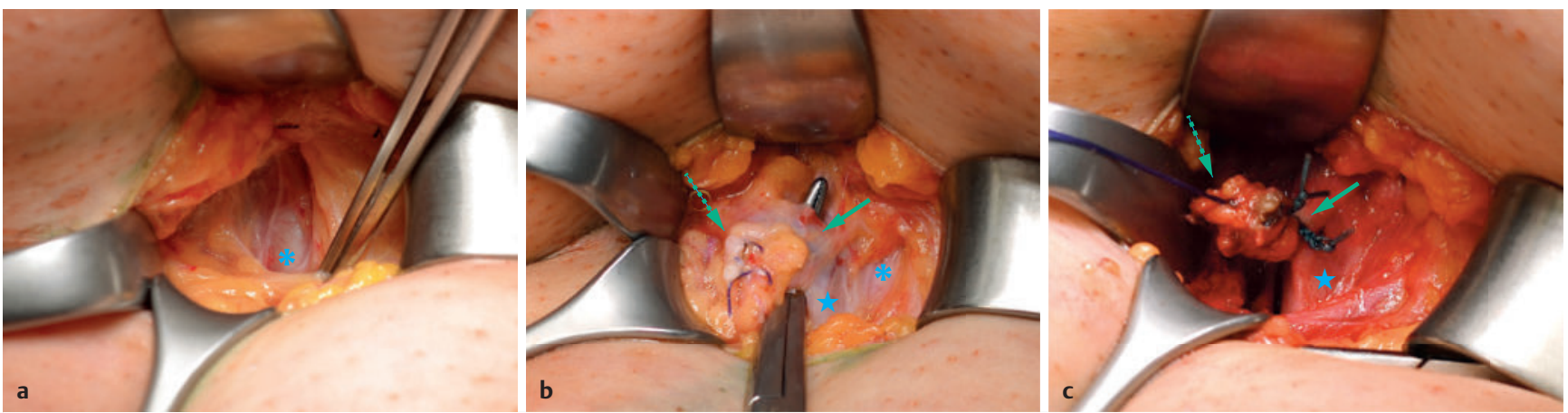

Abb. 4 Operativer Ablauf einer links inguinalen Crossenrevision. a Lateraler Zugang (modifiziert nach Junod [35]) über die A. femoralis communis (*) unter Umgehung des Narbengewebes. b Präparation nach medial über die V. femoralis communis (grobes Sternchen), Darstellung und Unterfahren des Saphena-Stumpfes (Pfeil) mit Overholt-Klemme. Das Narbengewebe (gestrichelter Pfeil) wird so bis zum Absetzen am saphenofemoralen Übergang nicht wesentlich tangiert. c Situs nach doppelter Stumpfligatur im Niveau der V. femoralis communis mit nicht resorbierbarem Nahtmaterial (Pfeil) und invertierender Endothelinversionsnaht (gestrichelter Pfeil). Eine präzise Beschreibung der OP-Technik findet sich in [15].

- Tab. 2 Studien zur operativen Therapie des inguinalen Crossenrezidivs.

\begin{tabular}{|c|c|c|c|c|c|c|c|}
\hline $\begin{array}{l}\text { Erstautor und } \\
\text { Publikationsjahr }\end{array}$ & $\begin{array}{l}\text { Studien- } \\
\text { typ }\end{array}$ & Studienarme & $\begin{array}{l}\text { Anzahl } \\
\text { (Beine) }\end{array}$ & $\begin{array}{l}\text { FU } \\
\text { (Mo) }\end{array}$ & $\begin{array}{l}\text { FU-Rate } \\
\text { (\%) }\end{array}$ & $\begin{array}{l}\text { Duplexsono- } \\
\text { graphisches ICRR }\end{array}$ & $\begin{array}{l}\text { Klinisches } \\
\text { ICCR }\end{array}$ \\
\hline Bhatti 2000 [36] & PK & ICR + PTFE-Patch & 81 & 19 & 86 & $37 \%$ & $12 \%$ \\
\hline Creton 2002 [38] & PK & ICR + PTFE-Patch & 170 & 59 & 70 & $13 \%$ & $4 \%$ \\
\hline Winterborn 2007 [39] & RKS & ICR + PTFE-Patch & 40 & 24 & 80 & $31 \%$ & $13 \%$ \\
\hline Freis 2016 [14] & RS & ICR + PTFE-Patch & 86 & 12 & n. a. & $?$ & $2 \%$ \\
\hline De Maeseneer 2004 [13] & nRKS & ICR + Silikon-Patch & 73 & 60 & 93 & $9 \%$ & $\begin{array}{l}26 \% \text { („thigh } \\
\text { varicosities“) }\end{array}$ \\
\hline Gerontopoulou 2018 [15] & FUS & ICR + Endothelinversionsnaht & 100 & 18 & n. a. & $5 \%$ & $3 \%$ \\
\hline
\end{tabular}

für die Patienten $[8,16,18]$. Interessant ist aber, dass sich letzteres nicht negativ auf die Zufriedenheit mit der Behandlung auswirkt $[14,15,36]$. Trotz geringerer Komplikationsrate nach EVLA im Vergleich zur offenen Operation für die Behandlung inguinaler Crossenrezidive zeigte sich in der oben bereits erwähnten Studie kein Unterschied in der postoperativen Patientenzufriedenheit [34].

Dennoch muss man zur Kenntnis nehmen, dass auch nach einer sorgfältig durchgeführten inguinalen Crossenrevision eine erneute Neovaskularisation und in der Folge klinisch relevante CrossenRe-Rezidive auftreten können. Historisch lag die Rate duplexsonographischer Crossen-Re-Rezidive bei ca. 70\% nach 2,5 Jahren; also ein nahezu wirkungsloser therapeutischer Ansatz [37]. Bis heute konnte diese Zahl jedoch durch diverse Maßnahmen drastisch reduziert werden. Um das Risiko der Neovaskularisation zu reduzieren wurden in den letzten 20 Jahren insbesondere anatomische oder prothetische Barrieretechniken, wie z. B. die Endothelinversionsnaht des Crossenstumpfes, der nahttechnische Verschluss der Fascia cribrosa oder das Einnähen eines Silikon- oder Polytetrafluoroethylene(PTFE)-Patches, entwickelt und in den Operationsablauf der inguinalen Crossenrevision integriert [13,15].
Zum Einsatz von Barrieretechniken in der inguinalen Revisionschirurgie sind in den vergangenen Jahren mehrere prospektive Kohortenstudien, einige retrospektive Studien, randomisierte und nicht-randomisierte kontrollierte Studien publiziert worden. Ein Überblick gibt $>$ Tab. 2. Nach Anwendung eines PTFE-Patches zeigen sich in der Literatur duplexsonographische Re-Rezidivraten von $13-37 \%$ und klinische inguinale Crossen-Re-Rezidive bei $2-13 \%$ der Patienten nach Nachbeobachtungszeiten von 12-59 Monaten $[14,36,38,39]$. De Maeseneer et al. untersuchten die Anwendung eines Silikon-Patches in einer prospektiven nicht-randomisierten Vergleichsstudie. Die mit Silikon-Patch versorgte Patientengruppe zeigte nach 5 Jahren v. a. eine um 80 \% reduzierte duplexsonographische Re-Rezidiv-Rate gegenüber der Nicht-Patch-Gruppe ( $9 \%$ vs. $45 \%$ ) [13]. Mit einer einfachen Barrieremaßnahme, der Endothelinversionsnaht nach Frings [40] ( $\mathbf{A b b}$. 4c), konnte unsere Arbeitsgruppe eine duplexsonographische Re-Rezidivrate von $5 \%$ und klinische inguinale Crossen-Re-Rezidive bei nur $3 \%$ der Patienten nach 18 Monaten Nachbeobachtungszeit nachweisen [15]. Dieser Überblick zeigt, dass die Anwendung von Barrierestrategien im Rahmen der inguinalen Crossenrevision ein hohes Potenzial aufweist, 
um Neovaskularisationsraten und damit die Rate an klinisch relevanten Re-Rezidiven deutlich zu reduzieren.

\section{FAZIT}

Die vorhandenen jüngeren Studien weisen darauf hin, dass die offene Re-Crossektomie, von Spezialisten durchgeführt und mit Barrieretechniken verbunden, zunehmend gute Ergebnisse im Hinblick auf eine langfristige Rezidivfreiheit der saphenofemoralen Region bei geringen Komplikationsraten gewährleistet. Die technische Entwicklung der Operationstechnik ist noch nicht abgeschlossen, Details im operativen Vorgehen müssen weiter in Studien untersucht werden. Die inguinale Crossenrevision ist zeitgemäß, bei kaliberstarken Crossenstümpfen vermutlich die beste Therapieoption und gehört mit den endovenösen Verfahren und der Schaumsklerosierung fest in das Behandlungsspektrum des saphenofemoralen Rezidivs.

Dennoch bleibt die Rezidivvarikose eine therapeutische Herausforderung, auch aufgrund des noch unzureichenden Wissens zu Ätiologie und Pathogenese [25]. Mit den endovenös thermischen Ablationsverfahren und der ultraschallgesteuerten Schaumsklerosierung stehen im Vergleich zur offenen Crossenrevision weniger invasive Therapieverfahren zur Verfügung, die entsprechend zunehmend zum Einsatz kommen. Die Studienlage ist jedoch insgesamt dürftig mit kurzen Nachbeobachtungszeiten und teils geringen Patientenzahlen. Es fehlt absolut an vergleichenden Studien der drei genannten Therapieverfahren. Da die anatomischen Befunde sehr unterschiedlich sein können, muss hier an präzisen Beschreibungen und Klassifikationssystemen gearbeitet werden. Vor dem Hintergrund, dass unterschiedliche Befunde vermutlich durch unterschiedliche Verfahren optimal behandelt werden können ( $\triangleright$ Abb. 1.), sollte durch entsprechende Studien eine Individualisierung der Therapie in den Fokus rücken. Um in der Zukunft mehr Evidenz zu schaffen und entsprechende Empfehlungen einer differenzierten Therapie festlegen zu können, brauchen wir eine bessere Studienlage.

\section{Interessenkonflikt}

Frau Gerontopoulou: Reisekosten- und Kongressbesuchsunterstützung durch Bauerfeind AG

\section{Literatur}

[1] Rabe E, Guex J], Puskas A, et al. VCP Coordinators. E\}pidemiology of chronic venous disorders in geographically diverse populations: results from the Vein Consult Program. Int Angiol 2012; 31: 105-115

[2] Darvall KA, Bate GR, Adam D], et al. Generic health-related quality of life is significantly worse in varicose vein patients with lower limb symptoms independent of CEAP clinical grade. Eur J Vasc Endovasc Surg 2012; 44: 341-344

[3] Chang SL, Huang YL, Lee MC Hu S et al. Association of varicose veins with incident venous thromboembolism and peripheral artery disease. JAMA 2018; 319: 807-817
[4] Kluess HG, Noppeney T, Breu FX et al. Leitlinie zur Diagnostik und Therapie der Krampfadererkrankung - Entwicklungsstufe S2. Phlebologie 2010; 39: 271-289

[5] NICE clinical guideline 168.Varicose veins in the legs - the diagnosis and management of varicose veins. Issued: July 2013. http://guidance. nice.org.uk/CG168

[6] Wittens C, Davies AH, Bäkgaard N et al. Management of Chronic Venous Disease; Clinical Practice Guidelines of the European Society for Vascular Surgery (ESVS). Eur J Vasc Endovasc Surg 2015; 49: 678-737

[7] Rass K, Frings N, Glowacki P et al. Same site recurrence is more frequent after endovenous laser ablation compared with high ligation and stripping of the great saphenous vein - 5-year results of a randomized clinical trial (RELACS study). Eur J Vasc Endovasc Surg 2015; 50: 648-656

[8] Perrin MR, Guex J], Ruckley CV et al. Recurrent varices after surgery (REVAS), a consensus document. REVAS group. Cardiovasc Surg 2000; 8: 233-245

[9] Rass K. Crossektomie und Stripping vs. endothermische Ablation der V. saphena magna: Was können wir aus aktuellen Langzeitanalysen lernen? Phlebologie 2018; 47: 265-271

[10] Theivacumar NS, Darwood R, Gough MJ. Neovascularization and recurrence 2 years after varicose vein treatment for sapheno-femoral and great saphenous vein reflux: a comparison of surgery and endovenous laser ablation. Eur J Vasc Endovasc Surg 2009; 38: 203-207

[11] De Maeseneer MG, Vandenbroeck CP, Hendriks JM et al. Accuracy of duplex evaluation one year after varicose vein surgery to predict recurrence at the sapheno-femoral junction after five years. Eur J Vasc Endovasc Surg 2005; 29: 308-312

[12] Geier B, Hummel T, Burger P. Residual stumps as a cause for inguinal varicose vein recurrences: A multicentre study. Eur J Vasc Endovasc Surg 2008; 36(2):207-210

[13] De Maeseneer MG, Vandenbroeck CP, Van Schil PE, Silicone patch saphenoplasty to prevent repeat recurrence after surgery to treat recurrent saphenofemoral incompetence: Long-term follow-up study. J Vasc Surg 2004; 40(1):98-105

[14] Freis $\mathrm{H}$, Geier B, Mumme A et al. Barrier patch implantation during redo surgery for varicose vein recurrences in the groin: 1 -year results. Ann Vasc Surg 2016; 35: 98-103

[15] Gerontopoulou SA, Kath W, Rass K. Short-Term Efficacy of Inguinal Reoperation for Recurrent Saphenofemoral Incompetence using the Stump Suture Technique. Ann Vasc Surg 2018;pii: S08905096(18)30432-1. doi: 10.1016/j.avsg.2018.04.015

[16] De Maeseneer M. Surgery for recurrent varicose veins: toward a less- invasive approach? Perspect Vasc Surg Endovasc Ther 2011; 23(4):244-249

[17] Creton D, Uhl JF. Foam sclerotherapy combined with surgical treatment for recurrent varicose veins: short term results. Eur J Vasc Endovasc Surg 2007; 33: 619-624

[18] Kostas T, Ioannou CV, Touloupakis E et al. Recurrent varicose veins after surgery: a new appraisal of a common and complex problem in vascular surgery. Eur J Vasc Endovasc Surg 2004; 27: 275-282

[19] De Maeseneer M, Pichot O, Cavezzi A et al. Duplex ultrasound investigation of the veins of the lower limbs after treatment for varicose veins e UIP consensus document. Eur J Vasc Endovasc Surg 2011; 42: 89-102

[20] Hamann S, Giang J, De Maeseneer M et al. Editor's Choice- Five Year Results of Great Saphenous Vein Treatment: A Meta-analysis. Eur J Vasc Endovasc Surg 2017; 54: 760-770

[21] Ellinghaus E, Ellinghaus D, Krusche P et al. Genome-wide association analysis for chronic venous disease identifies EFEMP1 and KCNH8 as susceptibility loci. Scientific RepoRts | 7: 45652 | DOI: 10.1038| srep45652 
[22] Pfisterer L, König G, Hecker M and Korff T. Pathogenesis of varicose veins - lessons from biomechanics. Vasa 2014; 43: 88-99

[23] Hartmann K, Klode J, Pfister R et al. Recurrent varicose veins: sonography-based re-examination of 210 patients 14 years after ligation and saphenous vein stripping. Vasa. 2006; 35(1):21-26

[24] Stücker M, Netz K, Breuckmann F et al. Histomorphologic classification of recurrent saphenofemoral reflux. J Vasc Surg 2004; 39: 816-821

[25] Brake M, Lim CS, Shepherd AC et al. Pathogenesis and etiology of recurrent varicose veins. J Vasc Surg 2013; 57: 860-868

[26] Blomgren L, Johansson G, Emanuelsson L et al. Late follow-up of a randomized trial of routine duplex imaging before varicose vein surgery. Br J Surg 2011; 98: 1112-1116

[27] Mumme A, Hummel T, Burger $P$ et al. High ligation of the saphenofemoral junction is necessary. Results of the German Groin Recurrence Study. Phlebologie 2009; 38: 99-102

[28] Theivacumar NS, Gough M]. Endovenous laser ablation (EVLA) to treat recurrent varicose veins. Eur J Vasc Endovasc Surg 2011; 41: 691-696

[29] Hwang JH, Park SW, Chang IS et al. Endovenous thermal ablation of recurrent varicose veins due to residual great saphenous venous insufficiency after saphenous venous surgery: A comparative study. Dermatol Surg 2018. doi: 10.1097/DSS.0000000000001543. [Epub ahead of print]

[30] Nwaejike N, Srodon PD, Kyriakides C. Endovenous laserablation for the treatment of recurrent varicose vein disease- a single centre experience. Int J Surg 2010; 8: 299-301

[31] Rabe E, Breu F X, Cavezzi A et al. for the Guideline Group: European guidelines for sclerotherapy in chronic venous disorders. Phlebology 2013; 29: 338-354
[32] Pavei P, Ferrini M, Spreafico G et al. Ultrasound guided foam sclerotherapy of recurrent varices of the great and small saphenous vein: 5 -year follow up. Veins and Lymphatics 2014; 3(4655):57-59

[33] Shepherd AC, Gohel MS, Lim CS et al. The treatment of varicose veins: an investigation of patient preferences and expectations. Phlebology 2010; 25: 54-65

[34] van Groenendael L, van der Vliet JA, Flinkenflögel L et al. Treatment of recurrent varicose veins of the great saphenous vein by conventional surgery and endovenous laser ablation. J Vasc Surg. 2009; 50(5):1106-1113

[35] Bruning G, Schinagl H. Surgical management of recurrent insufficiency of the sapheno-femoral junction using modified technique of Junod. J Dtsch Dermatol Ges 2011; 9: 646-647

[36] Bhatti TS, Whitman B, Harradine K et al. Causes of re- recurrence after polytetrafluoroethylene patch saphenoplasty for recurrent varicose veins. Br J Surg 2000; 87(10):1356-1360

[37] Gibbs PJ, Foy DM, Darke SG. Reoperation for recurrent saphenofemoral incompetence: a prospective randomized trial using a reflected flap of pectineus fascia. Eur J Vasc Endovasc Surg 1999; 18: 494-498

[38] Creton D. Surgery for recurrent Sapheno-femoral incompetence using expanded polytetrafluoroethylene patch interposition in front of the femoral vein: long term outcome in 119 extremities. Phlebology. 2002; 16: 93-97

[39] Winterborn R], Earnshaw JJ. Randomised Trial of Polytetrafluoroethylene Patch Insertion for Recurrent Great Saphenous Varicose Veins. Eur J Vasc Endovasc Surg 2007; 34: 367-373

[40] Frings N, Nelle A, Tran P et al. Reduction of neoreflux after correctly performed ligation of the saphenofemoral junction. A randomized trial. Eur J Vasc Endovasc Surg 2004; 28: 246-52 